\title{
Validación Colombiana del Inventario de Violencia en las Relaciones de Pareja en Adolescentes (CADRI)
}

\section{Colombian Validation of the Conflict in Adolescent Dating Relationships Inventory (CADRI)}

\author{
Jesús Redondo Pacheco ${ }^{1}$, Cesar Armando Rey Anacona ${ }^{2}$, Jaime Humberto Moreno Méndez ${ }^{3}$ y \\ Marianela Luzardo Briceño ${ }^{4}$
}

\begin{abstract}
Resumen
En los últimos años, la violencia de pareja se ha convertido en un problema de interés creciente por lo que es fundamental su correcta valoración en la población adolescente y joven. El objetivo es validar la versión en español del Conflict in Adolescent Dating Relationships Inventory (CADRI) en 2058 adolescentes colombianos con edades comprendidas entre los 12 y 19 años. El CADRI es un instrumento diseñado para evaluar cinco tipos de violencia en parejas de adolescentes y jóvenes: sexual, relacional, verbal-emocional, física y amenazas. Los resultados indican un ajuste adecuado tanto en la escala de victimización como en la de perpetración. Respecto a los índices de fiabilidad, se hallaron datos similares a los encontrados por los autores del instrumento original y en su versión española, con una consistencia interna adecuada. Como conclusión, se podría destacar que la versión colombiana del CADRI parece ser válida y fiable.
\end{abstract}

Palabras clave: validación, CADRI, adolescencia, violencia de pareja, medidas

\begin{abstract}
In the last few years, intimate partner violence has became a growing interest problem, which is why is essential the correct assessment in the adolescent and young population. The purpose is to validate the Spanish version of the Conflict in Adolescent Dating Relationships Inventory (CADRI) in 2058 Colombian adolescents aged between 12 and 19 years old. The CADRI is an instrument designed to evaluate five types of violence in adolescents and young adults: sexual, relational, verbal-emotional, physical, and threats. The results indicate an adequate adjustment in both the victimization and perpetration scales. Regarding the reliability indices, similar data was found by the authors of the original instrument and in its Spanish version with an adequate internal consistency. In conclusion, it could be emphasized that the Colombian version of the CADRI seems to be valid and reliable.
\end{abstract}

Keywords: validation, CADRI, adolescence, intimate partner violence, measures

\footnotetext{
${ }^{1}$ Doctor en Psicología. Universidad Pontificia Bolivariana - Bucaramanga, Colombia. Docente Investigador. Tel.: (+57)3155109410. Correo: jesus.redondo@upb.edu.co (autor de correspondencia)

${ }^{2}$ Doctor en Psicología Clínica y de la Salud. Universidad Pedagógica y Tecnológica de Colombia. Docente Investigador. Tel.: (+57) 3125890866. Correo: cesar.rey@uptc.edu.co

3 Doctor en Psicología. Universidad Católica de Colombia, Colombia. Docente Investigador. Tel.: +57 3002177911. Correo: jhmoreno@ucatolica.edu.co

${ }^{4}$ Doctora en Estadística. Universidad Pontificia Bolivariana - Bucaramanga, Colombia. Docente Investigadora. Tel.: (+57)3043370014. Correo: marianela.luzardo@upb.edu.co
} 


\section{Introducción}

La adolescencia es un periodo del desarrollo marcado por cambios importantes en las relaciones sociales con iguales y compañeros del sexo opuesto. Aunque en muchas de esas relaciones adolescentes se dan comportamientos saludables también ocurren otros de carácter coercitivo y agresivo con más frecuencia de lo esperado.

A lo largo de los últimos años se han llevado a cabo algunas revisiones sistemáticas en la literatura que buscaban conocer sobre la prevalencia e incidencia de este fenómeno, destacándose gran variabilidad entre los resultados (Jennings et al., 2017; Johnson, Parker, Rinehart, Nail, \& Rothman, 2015; Leen et al., 2013; Rubio-Garay, López-González, Carrasco, \& Amor, 2017; Shipley, 2018). Por ejemplo, respecto a la prevalencia en cuanto al sexo dicha variabilidad de resultados es mayor en el caso de la violencia física (Fernández-Fuertes, Fuertes, \& Pulido, 2006; Rey-Anacona, 2013). En ese sentido, se evidencia mayor prevalencia de violencia psicológica en mujeres (jugando los dos roles) (Esquivel-Santoveña, RodríguezHernández, Gutiérrez-Vega, Castillo-Viveros, \& López-Orozco, 2019; Jaén, Aragón, De Castro, \& Rivera, 2015; Rubio-Garay et al., 2017) y, en cuanto a violencia sexual, mayores tasas en agresiones de los hombres y de victimización en las mujeres (Contreras, Both, Guedes, \& Dartnall, 2016; Fernández-González, Calvete, \& Orue, 2020).

Así mismo, cabe señalar la existencia de violencia bidireccional, como una dinámica de pareja donde ambos miembros de la misma actúan como perpetradores y como víctimas (EsquivelSantoveña et al., 2019; Palmetto, Davidson, \& Rickert, 2013; Paradis, Hébert, \& Moreau, 2020; Rubio-Garay, López-González, Saúl, \& SánchezElvira-Paniagua, 2012), ya sea como un comportamiento de defensa (Duval, Lanning, \& Patterson, 2020) o como la agresión y respuesta entre los miembros de la pareja ante una situación determinada en contexto diferente (Palmetto et al., 2013). De cualquier forma, como ya se indicó anteriormente, los hombres utilizan más formas de violencia física frente al mayor sufrimiento físico y psicológico de las mujeres (Muñoz-Rivas,
Graña, O'Leary, \& González, 2007; Paradis et al., 2020; Terrazas-Carrillo \& Sabina, 2019).

Estos hallazgos mantienen la preocupación relacionada con que la adolescencia representa un período crítico y estos factores de riesgo asociados al comportamiento abusivo hacia la pareja se traducen en patrones duraderos de abuso en las actuales y futuras relaciones íntimas (Iglesias-García, Urbano-Contreras, \& MartínezGonzález, 2020; Jackson, Cram, \& Seymour, 2000; Redondo, Inglés, \& García, 2017). En este sentido, se trata de evento significativo que puede tener consecuencias inmediatas y tardías para la salud, además de ser un fuerte predictor de violencia conyugal en la edad adulta (ExnerCortens et al., 2016). Asimismo, varios estudios han encontrado asociación entre la violencia entre parejas adolescentes y diferentes problemas de salud como depresión, ansiedad y abuso de alcohol y otras drogas (Centers for Disease Control and Prevention, 2017; Taquette, \& Monteiro, 2019), conductas sexuales de riesgo, SIDA y bajo rendimiento académico (Barros \& Schraiber, 2017), entre otras.

En los últimos años ha habido un aumento significativo en la creación y adaptación de instrumentos que llevan a cabo una medida apropiada del abuso de parejas en adolescentes, considerando que existen comportamientos diferenciadores en las relaciones entre adultos y adolescentes, y destacando elementos que son inadecuados para los adolescentes (por ejemplo, usar los hijos como táctica de amenaza y control o el abuso económico, entre otros). Además, cabe señalar que las relaciones íntimas entre adolescentes y adultos varían mucho respecto a duración, nivel de compromiso, experiencia previa, grado de intimidad sexual, causas y resoluciones de conflicto, entre otras.

Así, se encuentran algunos instrumentos a nivel internacional (Conflict Tactics Scale, CTS, de Straus, 1979, y su versión revisada de Straus, Hamby, Money-McCoy, \& Sugarman, 1996 Revised Conflict Tactics Scale, CTS2; Psychological Maltreatment Women Inventory, PMWI, Tolman, 1999) y dos validaciones de instrumentos en español: El Cuestionario de Violencia entre Novios-CUVINO (RodríguezFranco et al., 2010) que cuenta con 42 ítems y que genera 8 factores (desapego, humillación, 
agresión sexual, coerción, agresión física, violencia de género, castigo emocional y violencia instrumental) y el Conflict in Adolescent Dating Relationships Inventory, Conflict in Adolescent Dating Relationships Inventory, CADRI (Wolfe et al., 2001) en población española donde se utilizan 3 sub-escalas que evalúan las agresiones contra la pareja (17 ítems) y comportamientos de victimización (17 ítems). Las dos subescalas se agrupan en 6 factores, a saber, Violencia $y$ Victimización Relacional, Violencia $y$ Victimización verbal-emocional y Violencia y Victimización física (Fernández-Fuertes et al., 2006).

Actualmente se evidencia en la literatura diferentes cuestionarios y escalas, muy bien validados y con una estructura factorial satisfactoria (López-Cepero, Rodríguez, \& Rodríguez, 2015). La clasificación más frecuente que se encuentra en dicha literatura evidencia el maltrato en físico, abuso psicológico y sexual (Franco, López-Cepero, \& Díaz, 2009), pero en otras revisiones se ha encontrado que los resultados de los análisis de factores pocas veces concuerdan con esta estructura de factores (Esquivel-Santoveña, Lambert, \& Hamel, 2013).

En este orden de ideas, la revisión sistemática de los instrumentos disponibles ha llegado a la conclusión de que una cuarta parte de los estudios de validación no proporcionan información sobre la estructura del instrumento, solo el $15 \%$ realiza un estudio confirmatorio de la estructura de los factores y ese $10 \%$ no proporciona información sobre su consistencia interna (Exner-Cortens, Gill, \& Eckenrode, 2016a; Exner-Cortens et al., 2016b; López-Cepero, Rodríguez-Franco, \& RodríguezDíaz, 2015). En Iberoamérica, una revisión llevada a cabo por Yanez-Peñúnuri, HidalgoRasmussen y Chávez-Flores (2019) encontró evidencia de consistencia interna, validez de contenido y de constructo de los instrumentos revisados, presentando puntuaciones más altas el Cuestionario de Maltrato en el noviazgo $(C M N)$ y el Violence in Adolescents' Dating Relationships Inventory (VADRI en España y en México) y dos adaptaciones del mismo instrumento (Conflict Tactics Scale, CTS, en Brasil y en México), según criterios de Terwee et al. (2007).

Actualmente, en Colombia existen pocas medidas de violencia entre parejas para adolescentes creadas por investigadores colombianos (Lista de Chequeo de Experiencias de Maltrato en la Pareja Forma A; Rey-Anacona, 2006) o adaptaciones de cuestionarios en inglés o español como la Modified Conflicts Tactics Scale, CTS (Muñoz-Rivas et al., 2007) (EEUU y España), el Index Spouse Abuse, ISA (Hudson \& McIntosh, 1981) (EEUU, Brasil, El Salvador, Perú y España), el Cuestionario de Violencia de Novios, CUVINO (Rodríguez-Franco et al., 2010) (EEUU, Argentina, México y España), y el mismo CADRI (Canadá, México y España).

El Inventario de Violencia en las Relaciones de Pareja en Adolescentes (Conflict in Adolescent Dating Relationships Inventory, Conflict in Adolescent Dating Relationships Inventory, CADRI) fue seleccionado para ser adaptado al contexto colombiano porque se trata de un instrumento bien desarrollado, ampliamente utilizado y una de las medidas de maltrato en la pareja más apoyadas empíricamente (Cascardi, Blank, \& Dodani, 2016; Exner-Cortens et al., 2016a; Toplu Demirtaş et al., 2018). El CADRI fue elaborado por Wolfe et al. (2001) considerando la forma como los adolescentes y jóvenes construyen sus relaciones de noviazgo y las posibles formas de violencia que se presentan y que permiten diferenciar los sujetos violentos y los no violentos. Está compuesto por 25 ítems, cada uno conformado por dos escalas, una relacionada con la conducta referida a agresiones perpetradas y la otra respecto a conductas violentas sufridas en momentos de discusión de la pareja. El CADRI evidencia cinco tipos de violencia: Violencia Verbal-Emocional, Violencia Sexual, Violencia Física, Amenazas y Violencia Relacional.

Las propiedades psicométricas del CADRI han demostrado ser satisfactorias en muestras de adolescentes españoles (Fernández-Fuertes et al., 2006) y mexicanos (Hokoda et al., 2006). Benítez y Muñoz (2005) realizaron un análisis factorial confirmatorio y establecieron un modelo de victimización y otro de perpetración valores adecuados de ajuste e índices elevados de consistencia interna.

Si bien el CADRI ya ha sido validado en población hispanohablante (Hokoda et al., 2006; Fernández-Fuertes et al., 2006), aún faltan adaptaciones para países latinoamericanos. Sería 
un error asumir la permanencia de las garantías psicométricas de la validación de España y México en el resto de países hispanohablantes. Aplicar el CADRI sin tomar en cuenta variables culturales entre países, podría implicar que los datos obtenidos no reflejan realmente la realidad de los adolescentes, sino la discrepancia en la comprensión de la violencia en el noviazgo adolescente mediada por variables culturales y temporales como la religión, estilo de vida y valores.

Por ejemplo, se dice que los latinos tienen actitudes más tradicionales sobre las mujeres, las relaciones y el compromiso, y los colombianos pueden tener expectativas más rígidas sobre los roles de género que las poblaciones norteamericanas o europeas. Aunque este tipo de creencias están cambiando y pueden variar entre grupos urbanos y rurales, es importante la influencia subjetiva de estas creencias sobre la medida de la violencia en el noviazgo (RonzónTirado, Muñoz-Rivas, Zamarrón-Cassinello, \& Redondo-Rodríguez, 2019; Shaffer et al., 2018).

Además, al realizar estudios comparativos transculturales, las variantes encontradas pueden no mostrar las similitudes o diferencias entre países, sino las deficiencias del CADRI al evaluar cada población mediada por el uso de la lengua, tales como, estructura familiar del lenguaje o la equivalencia semántica (Martínez-Dorado et al., 2020).

Dada la importancia de contar con una medida psicométricamente sólida para evaluar la violencia entre las parejas de jóvenes, el propósito de esta investigación es examinar las evidencias de validez y confiabilidad de las puntuaciones del CADRI en el contexto colombiano.

\section{Método}

\section{Participantes}

Los participantes fueron seleccionados a través de un muestreo no aleatorio, según su disponibilidad en las instituciones participantes, contando finalmente con una muestra de 2058 adolescentes con edades entre los 12 y los 19 años $(M=16.15, D T=1.61)$, de los cuales 1088 eran mujeres (52.86\%) y 970 varones $(47.13 \%)$ y que cursaban bachillerato en instituciones de educación media de Bogotá $(n=738)$,
Bucaramanga $(\mathrm{n}=323)$, Tunja $(\mathrm{n}=495)$ y Yopal $(\mathrm{n}=502)$. Los participantes tuvieron, en promedio, cuatro relaciones de pareja, con una duración media de 6.86 meses en la relación actual y 7.51 meses en la relación anterior.

Los criterios de inclusión y exclusión que se consideraron fueron: (a) Tener o haber tenido una relación amorosa de al menos un mes de duración; (b) tener en el momento de la investigación la edad de 12 a 19 años; (c) no estar casado(a) ni convivir con su pareja; y (d) consentimiento de los padres y asentimiento del adolescente firmado (excepto los mayores de edad).

\section{Instrumento}

CADRI, versión española (Fernández-Fuertes et al., 2006). Se trata de un instrumento creado por Wolfe et al. (2001) que informa sobre la agresión y victimización de comportamientos relacionados con cinco tipos de violencia: Violencia VerbalEmocional (10 ítems), Violencia Sexual (4 ítems), Violencia Física (4 ítems), Amenazas (4 ítems) y Violencia Relacional (3 ítems). Las respuestas se llevan a cabo mediante una escala tipo Likert que va desde 0 (nunca ha pasado en nuestra relación); hasta 3 (con frecuencia- se ha producido en 6 o más ocasiones). Además, el instrumento cuenta con otros 20 ítems relacionados con una adecuada solución de conflictos de pareja, que no fueron tenidos en cuenta en esta investigación para evitar que la duración de la aplicación de los instrumentos fuera excesiva. Wolfe et al. (2001) destacan una estructura donde sus 25 ítems son distribuidos en cinco factores de primer orden: Violencia sexual $(\alpha=.51)$, Violencia relacional $(\alpha=.52)$, Violencia verbal-emocional ( $\alpha=.82)$, Amenazas $(\alpha=.66)$ y Violencia física $(\alpha=.83)$. Después de ser refactorializadas, estas dimensiones configuran un factor de segundo orden llamado Violencia. Por último, los autores de la prueba original señalan un coeficiente de estabilidad de $.68(p<.001)$ a través de un test-retest.

En este sentido, Fernández-Fuertes et al. (2006) aplicaron el instrumento a 572 estudiantes españoles, entre 15 y 19 años de edad, realizando un análisis factorial exploratorio de componentes principales con índices de consistencia interna entre .51 (en la escala de Amenazas) y .79 (en la escala de Violencia Verbal-Emocional), con un valor alfa general de .86 para todo el cuestionario. 


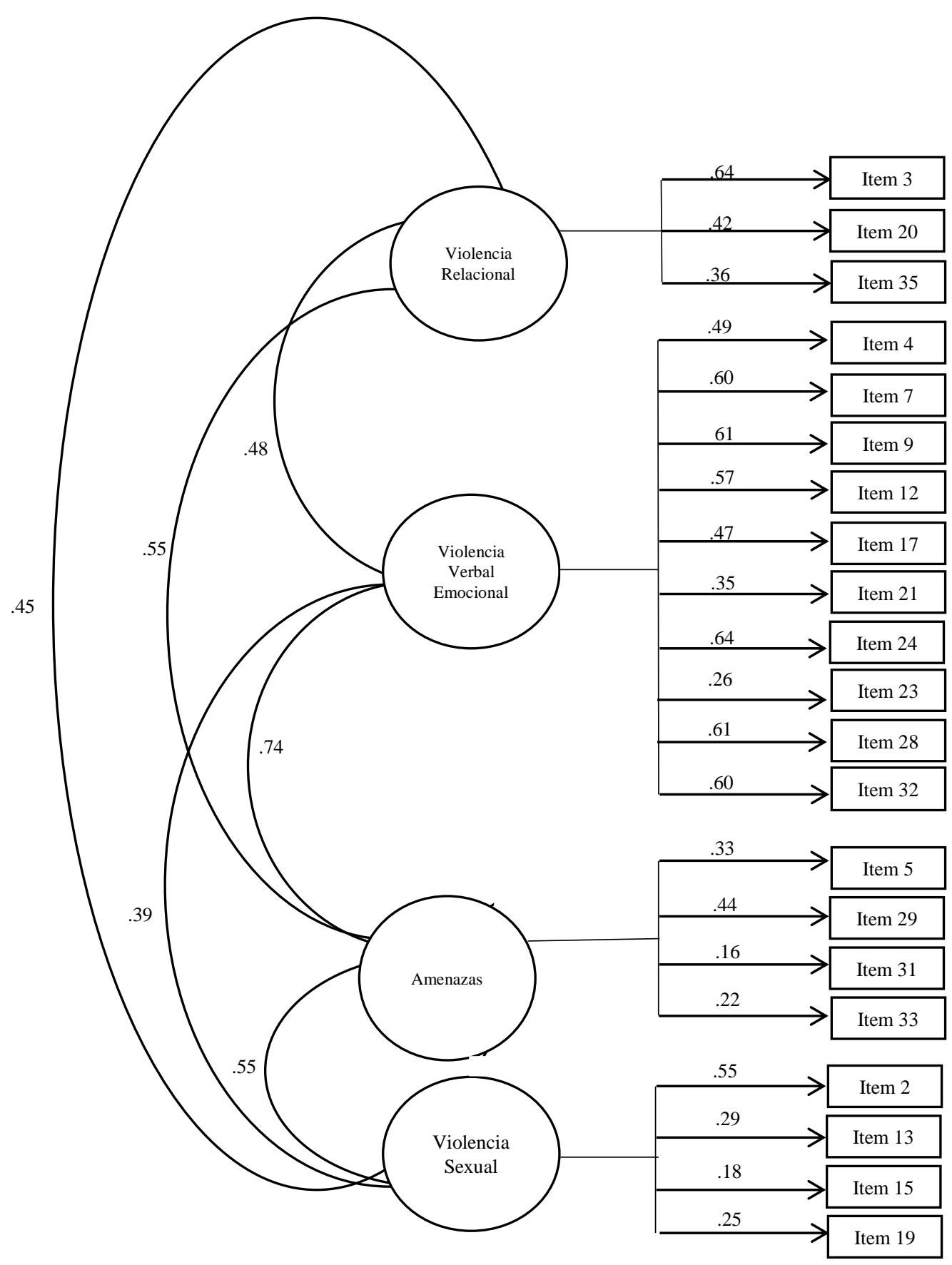

Figura 1. Parámetros Estimados Estandarizados para el Modelo de Medida de la escala de victimización del CADRI

\section{Procedimiento}

El procedimiento con los participantes se llevó a cabo en tres fases. En una primera fase se hizo una presentación a las directivas de las instituciones solicitando los permisos correspondientes. La segunda fase estaba relacionada con la solicitud del consentimiento informado a los padres y el asentimiento informado a los estudiantes y la posterior aplicación del instrumento en el colegio. Los participantes conocían el objetivo del estudio además de que su participación era voluntaria y anónima, y que podían abandonar en cualquier momento. Finalmente, en la tercera fase se llevaron a cabo los análisis de resultados y la discusión. 
Tabla 1. Consistencia interna y medidas descriptivas de la escala de victimización del CADRI

\begin{tabular}{lccc}
\hline & $M$ & $D T$ & $\alpha$ \\
\hline Violencia física & 0.57 & 1.36 & .74 \\
Violencia sexual & 1.20 & 1.67 & .56 \\
Violencia relacional & 0.79 & 1.46 & .73 \\
Violencia verbal emocional & 6.76 & 5.47 & .83 \\
Amenazas & 0.72 & 1.28 & .69 \\
Total & 10.09 & 8.90 & .84 \\
\hline
\end{tabular}

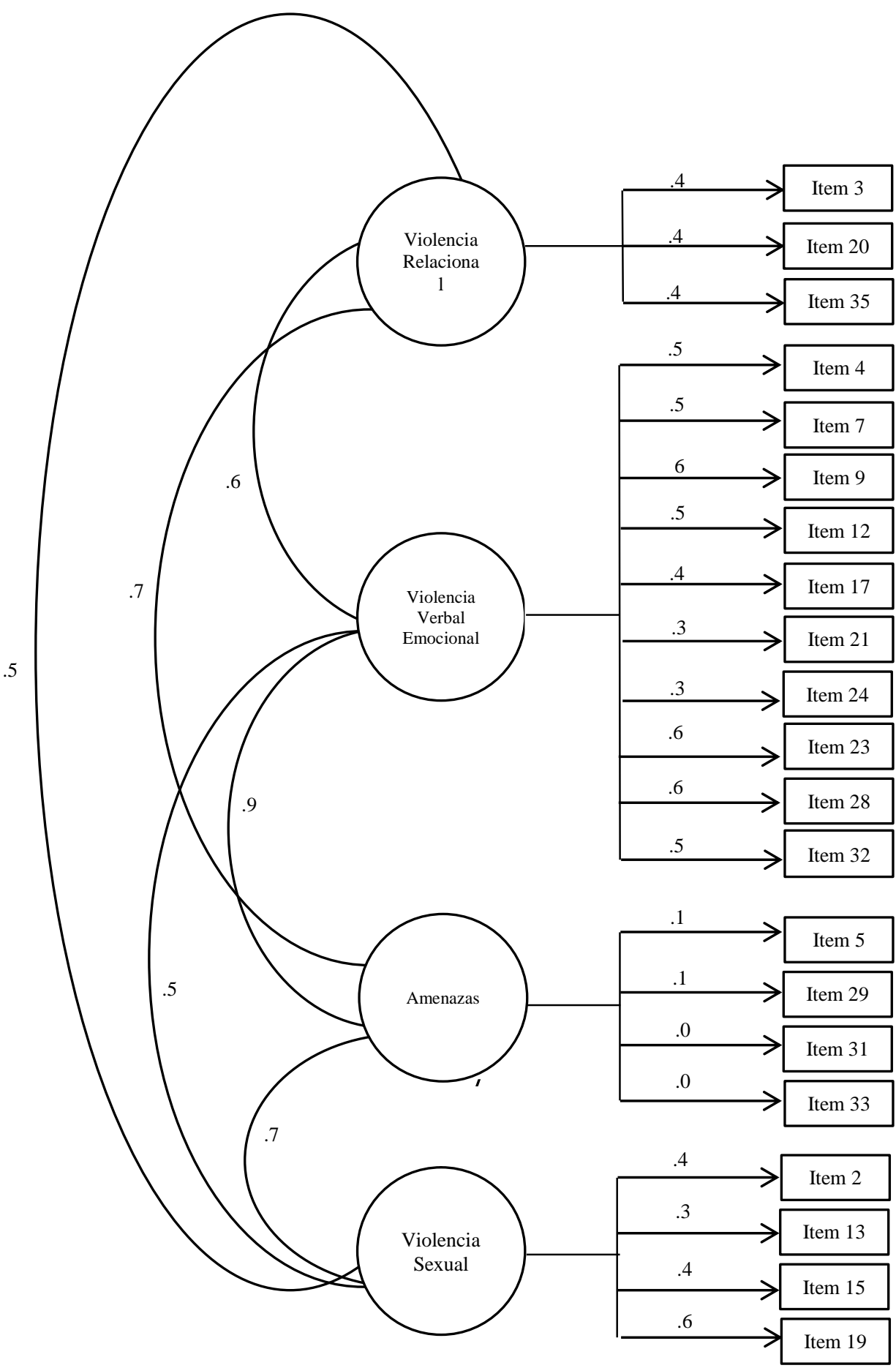

Figura 2. Parámetros Estimados Estandarizados para el Modelo de Medida de la escala de perpetración del CADRI

En la presente investigación se tuvieron en cuenta los principios éticos de experimentación com humanos considerándose de riesgo mínimo según el artículo 11 del apartado $\mathrm{B}$ de la Resolución 8430 de 1993 (Ministerio de Salud, Republica de Colombia, 1993). 
Tabla 2. Consistencia interna y medidas descriptivas de las escala perpetración del CADRI

\begin{tabular}{lccc}
\hline & $M$ & $D T$ & $\alpha$ \\
\hline Violencia física & 0.62 & 1.51 & .75 \\
Violencia sexual & 1.20 & .73 & .67 \\
Violencia relacional & 0.79 & 1.46 & .74 \\
Violencia verbal emocional & 6.77 & 5.45 & .83 \\
Amenazas & 0.72 & 1.28 & .72 \\
Total & 10.09 & 8.90 & .73 \\
\hline
\end{tabular}

\section{Análisis estadístico}

Mediante el software Amos, versión 6, se implementó un análisis factorial confirmatorio (CFA) midiendo el ajuste robusto del modelo a través del TLI, CFI (obteniendo un buen ajuste con valores >.90) y RMSEA (valores <.08 para un buen ajuste) (Bentler, 1995) utilizando la estimación de máxima verosimilitud.

Por otro lado, se análizó a partir del alfa de Cronbach la consistencia interna para las escalas de victimización y perpetración.

\section{Resultados}

En cuanto a la escala de victimización del CADRI, se estimó el modelo mediante máxima verosimilitud obteniendo que todos los ítems fueron significativos, encontrando un ajuste adecuado después de intervenir algunos índices de modificación, obteniendo una $\chi^{2}=864.647$; $\mathrm{gl}=462, p<.001$ para un cociente de $1.87<2$, lo que indica que el ajuste es bueno. Por otro lado, se lograron índices que señalan un buen ajuste del modelo estimado tales como: AGFI=.903 (.901.00); GFI=.913 (.90-.95) y RMSEA=.015 (0-.05). Este modelo también fue estimado por el método de mínimos cuadrados siendo el de máxima verosimilitud el que menos $A I C$ obtuvo en la estimación: $\mathrm{AIC}=1140$ (MV) versus 2810 (MC).

En la Figura 1 se presentan los parámetros estandarizados del modelo final. La consistencia interna para las escalas osciló entre .56 y .84 (alfa de Cronbach). Además, en la Tabla 1 se evidencia la información descriptiva de la escala de victimización del CADRI, destacando que, respecto a la victimización, la violencia verbal emocional y sexual son las más comunes, mientras que los otros tipos de violencia presentan menores puntuaciones medias.

Asimismo, respecto a la escala de perpetración del CADRI, a través de la estimación del modelo mediante máxima verosimilitud se encontró que todos los ítems también fueron significativos, con un ajuste adecuado después de intervenir algunos índices de modificación $\left(\chi^{2}=877.568 ; \mathrm{gl}=456, p<.001\right.$ para un cociente de $1.89<2)$. Además, los índices señalan un buen ajuste del modelo estimado (AGFI=.908 (.90$1.00)$; GFI=.907 (.90-.95) y RMSEA=.015 (0-.05). A través del método de mínimos cuadrados el modelo de máxima verosimilitud fue el que menos AIC obtuvo en la estimación: $\mathrm{AIC}=1165.56 \quad$ (MV) versus $2810 \quad$ (MC) (ver Figura 2).

La consistencia interna para las cuatro escalas de perpetración osciló entre .72 y .83 (alfa de Cronbach) y su información descriptiva señala que, al igual que en el caso de la escala de victimización, la violencia verbal emocional y la sexual son las que presentan mayores puntuaciones medias frente a los otros tipos de violencia (ver Tabla 2).

\section{Discusión}

Según la Organización Mundial de la SaludOMS (2015), la violencia entre las parejas se trata de un serio problema de salud pública. Este tipo de violencia presenta una fuerte naturaleza de género, ya que sus porcentajes más altos se evidencian en las mujeres en diferentes niveles: individual, de relación, en comunidad y en sociedad (Krantz \& García-Moreno, 2015). Existen diferentes investigaciones que muestran una brecha significativa entre la investigación con población adulta y adolescente/joven (Benítez, \& Muñoz, 2014; Duval et al., 2020). Por ello, son necesarias herramientas de evaluación desarrolladas específicamente para la población joven y adolescente en sus relaciones amorosas ya que existen diferencias notables entre los reportados para la población adulta y adolescente

Esta investigación aporta información sobre la estructura factorial y los índices de consistencia 
interna de la victimización y perpetración en las relaciones de pareja de los adolescentes a través del CADRI utilizando una muestra de 2058 participantes con edades comprendidas entre los 12 y 19 años de ambos sexos. Las cinco escalas del CADRI abarcan los tipos de violencia en pareja presentes en la literatura científica (Esquivel-Santoveña et al., 2013). Nuestros resultados apoyan la estructura factorial reportada en estudios previos en español (Fernández-Fuertes et al., 2006), lo que confirma un ajuste adecuado tanto de la escala de victimización como en la de perpetración, demostrando que se mantienen las estructuras factoriales subyacentes de Wolfe et al. (2001), a pesar de las diferentes condiciones sociales y culturales de los países. Esto nos lleva a pensar que sería muy importante un estudio transcultural para determinar la relación entre la violencia en el noviazgo y otras variables como rasgos de personalidad, estructura familiar, nivel educativo y consumo con sustancias psicoactivas, entre otras.

Además, los índices de consistencia interna de las escalas han sido superiores a los obtenidos en la validación con muestra española del CADRI (Fernández-Fuertes et al., 2006). Finalmente, los resultados de los análisis factoriales confirmatorios han demostrado que los 25 pares de ítems se agrupan en cinco dimensiones, tanto de victimización como de perpetración, teóricamente significativas: violencia física, verbal-emocional, relacional, sexual y amenazas.

Cabe señalar que el instrumento mide la violencia íntima entre personas que no están casadas o que no viven juntas. Pensamos que este aspecto podría permitirnos entender algunos efectos interesantes relacionados con la violencia en las parejas más jóvenes. Nuestros resultados también apuntan a la necesidad de revisión de algunos factores, especialmente en lo que respecta al relacionado con las herramientas de evaluación ya que se trata de técnicas dinámicas que requieren de revisiones continuas. En este sentido, es muy importante que se revisen aspectos relacionados con otros tipos de violencia, por ejemplo la instrumental o la económica, así como la violencia a través de las Tecnologías de Información y Comunicación (TICs).

El CADRI evalúa la violencia en la pareja teniendo en cuenta a una serie de indicadores que muestran su validez lo que podría facilitar el diseño e implementación de medidas de acción temprana, por lo que las implicaciones prácticas de este instrumento podrían ser variadas. A pesar de esto, los resultados obtenidos presentan ciertas limitaciones. Por ejemplo, respecto a la operativización de las variables, debido a las medidas de autoinforme que, en general, podrían estar sesgadas por la subjetividad y la deseabilidad social de los participantes, por tratarse de conductas agresivas y, más concretamente, cuando se trata de conductas violentas hacia la pareja por parte de adolescentes. Wolfe et al. (2001) discuten esta cuestión más a fondo respecto a las limitaciones del CADRI original, y enfatizan que sería importante aclarar los contextos y consecuencias de los episodios violentos en cada uno de los miembros de la pareja. Futuras investigaciones deberían confirmar los resultados obtenidos en este estudio utilizando muestras de otros contextos.

Teniendo en cuenta que la agresión en las relaciones de pareja en adolescentes y jóvenes es un problema importante debido a su alta prevalencia, si consideramos que las relaciones en esta edad pueden ser entendidas como un aprendizaje de roles, comportamientos y actitudes que fomentan la violencia en adultos, se justifica la relevancia de investigar la violencia en este período de vida para así poder desarrollar programas que ayudan a prevenir patrones de comportamiento violento (Durán \& RodríguezDomínguez, 2020; García-Díaz, Fernández-Feito, Bringas-Molleda, Rodríguez-Díaz, \& Lana, 2020; Makleff et al., 2020; Mérida, 2019). Está claro que se necesita un mayor número de estudios donde se exploren aspectos como las actitudes que apoyan la agresión como una solución justificable al conflicto entre parejas o las características de las relaciones anteriores, niveles de conciencia sobre el abuso de mujeres jóvenes o la relación entre los niveles de tolerancia al abuso y la frecuencia de actos violentos cometidos en la pareja, por citar algunos (Esquivel-Santoveña et al., 2019; Peskin et al., 2019; Terrazas-Carrillo \& Sabina, 2019). 


\section{Referencias}

Barros, C. R. D. S., \& Schraiber, L. B. (2017). Intimate partner violence reported by female and male users of healthcare units. Revista de Saúde Publica, 51, 7-17. https://doi.org/10.1590/S15188787.2017051006385

Benítez, J. L., \& Muñoz, J. F. (2014). Análisis factorial de las puntuaciones del CADRI en adolescentes universitarios españoles. Univetrsitas Psychologica, 12(1), 175-186. http://dx.doi.org/10.11144/Javeriana.UPSY131.afpc

Bentler, P. M. (1995). EQS structural equations program manual. Encino, CA: Multivariate Software.

Cascardi, M., Blank, S., \& Dodani, V. (2016). Comparison of the CADRI and CTS2 for measuring psychological and physical dating violence perpetration and victimization. Journal of Interpersonal Violence, 1-26. http://dx.doi.org/10.1177/0886260516670182

Centers for Disease Control and Prevention. (2017). Injury Prevention and Control Division of Violence Prevention Understanding Teen Dating Violence. CDC 2016. Retrieved from https://www.cdc.gov/violenceprevention/intim atepartnerviolence/teendatingviolence/fastfact .html

Contreras, J. M., Both, S., Guedes, A., \& Dartnall, E. (2016). Violencia sexual en Latinoamérica y el Caribe: Análisis de datos secundarios. Iniciativa de Investigación sobre la Violencia Sexual. Recuperado de http://clacaidigital.info/handle/123456789/980

Durán, M., \& Rodríguez-Domínguez, C. (2020). Social perception of situations of sexual cyberviolence: The role of sexist attitudes and the victim's transgression of gender roles. International Journal of Social Psychology, 35(1), 148-174. https://doi.org/10.1080/02134748.2019.16822 95

Duval, A., Lanning, B. A., \& Patterson, M. S. (2020). A systematic review of dating violence risk factors among undergraduate college students. Trauma, Violence \& Abuse, 21(3), 567-585. https://doi.org/10.1177/1524838018782207

Esquivel-Santoveña, E. E., Lambert, T. L., \& Hamel, J. (2013). Partner Abuse Worldwide. Partner Abuse, 4, 6-75. http://dx.doi.org/10.1891/1946-6560.4.1.6

Esquivel-Santoveña, E. E., Rodríguez-Hernández, R., Gutiérrez-Vega, M., Castillo-Viveros, N., \& López-Orozco, F. (2019). Psychological aggression, attitudes about violence, violent socialization, and dominance in dating relationships. Journal of Interpersonal Violence, 1-22.

https://doi.org/10.1177/0886260519842856

Exner-Cortens, D., Gill, L., \& Eckenrode, J. (2016). Measurement of adolescent dating violence: A comprehensive review (Part 1, behaviors). Aggression and Violent Behavior, 27, 64-78. https://doi.org/10.1016/j.avb.2016.02.007

Exner-Cortens, D., Gill, L., \& Eckenrode, J. (2016). Measurement of adolescent dating violence: A comprehensive review (Part 2, attitudes). Aggression and Violent Behavior, 27, 93-106.

http://dx.doi.org/10.1016/j.avb.2016.02.011

Fernández-Fuertes, A. A., Fuertes, A., \& Pulido, R. F. (2006). Evaluación de la violencia en las relaciones de pareja de los adolescentes. Validación del Conflict in Adolescent Dating Relationships Inventory (CADRI)-versión española. International Journal of Clinical and Health Psychology, 6(2), 339-358. Recuperado de https://www.redalyc.org/pdf/337/33760208.pd $\mathrm{f}$

Fernández-González, L., Calvete, E., \& Orue, I. (2020). Adolescent dating violence stability and mutuality: A 4-year longitudinal study. Journal of Interpersonal Violence, 35(9-10), 2012-2032.

https://doi.org/10.1177/0886260517699953

Franco, L. R., López-Cepero, J., \& Díaz, F. J. R. (2009). Violencia doméstica: Una revisión bibliográfica y bibliométrica. Psicothema, 21, 248-254. Recuperado de https://www.redalyc.org/pdf/727/7271165401 2.pdf

García-Díaz, V., Fernández-Feito, A., BringasMolleda, C., Rodríguez-Díaz, F. J., \& Lana, A. (2020). Tolerance of intimate partner 
violence and sexist attitudes among health sciences students from three Spanish universities. Gaceta Sanitaria, 34, 179-185. http://dx.doi.org/10.1016/j.gaceta.2019.01.003

Hokoda, A., Ramos-Lira, L., Celaya, P., Vilhauer, K., Angeles, M., Ruíz, S., Malcarne, V. L., \& Duque, M. (2006). Reliability of translated measures assessing dating violence among Mexican adolescents. Violence and Victims, 21, 117-127. http://dx.doi.org/10.1891/08866708.21.1.117

Hudson, W. W., \& McIntosh, S. R. (1981). The assessment of spouse abuse: Two quantifiable dimensions. Journal of Marriage and the Family, $\quad 43, \quad 873-885$. http://dx.doi.org/10.2307/351344

Iglesias-García, M. T., Urbano-Contreras, A., \& Martínez-González, R. A. (2020). Escala de Frecuencia y Respuesta a los Conflictos de Pareja: Fiabilidad y Validez. Revista Iberoamericana de Diagnóstico y Evaluación - e Avaliação Psicológica, 56(3), 47-57. https://doi.org/10.21865/RIDEP56.3.04

Jackson, S. M., Cram, F., \& Seymour, F. W. (2000). Violence and sexual coercion in high school students' dating relationships. Journal of Family Violence, 15(1), 23-36. Retrieved from

https://www.researchgate.net/profile/Fiona_Cr am2/publication/226797792_Violence_and_S exual_Coercion_in_High_School_Students'_ Dating_Relationships/links/5c81e309299bf12 68d44b01c/Violence-and-Sexual-Coercion-inHigh-School-Students-Dating-

Relationships.pdf

Jaén, C. I., Aragón, S. R., de Castro, E. F. A., \& Rivera, L. R. (2015). Violencia de pareja en mujeres: prevalencia y factores asociados. Acta de Investigación Psicológica, 5(3), 2224-2239. https://doi.org/10.1016/s2007-4719(16)30012-6

Jennings, W. G., Okeem, C., Piquero, A. R., Sellers, C. S., Theobald, D., \& Farrington, D. P. (2017). Dating and intimate partner violence among young persons ages 15-30: Evidence from a systematic review. Aggression and Violent Behavior, 33, 107-125.

https://doi.org/10.1016/j.avb.2017.01.007
Johnson, R. M., Parker, E. M., Rinehart, J., Nail, J., \& Rothman, E. F. (2015). Neighborhood factors and dating violence among youth: A systematic review. American Journal of Preventive Medicine, 49(3), 458-466. http://dx.doi.org/10.1016/j.amepre.2015.05.02 0

Krantz, G., \& García-Moreno, C. (2005). Violence against women. Journal of Epidemiology \& Community Health, 59(10), 818-821. http://dx.doi.org/10.1136/jech.2004.022756

Leen, E., Sorbring, E., Mawer, M., Holdsworth, E., Helsing, B., \& Bowen, E. (2013). Prevalence, dynamic risk factors and the efficacy of primary interventions for adolescent dating violence: An international review. Aggression and Violent Behavior, 18, 159-174. http://dx.doi.org/10.1016/j.avb.2012.11.015

López-Cepero, J., Rodríguez, L., \& Rodríguez, F. J. (2015). Evaluación de la violencia de pareja. Una revisión de instrumentos de evaluación conductual. Revista Iberoamericana de Diagnóstico y Evaluación - e Avaliação Psicológica, 2(40), 37-50. Recuperado de https://idus.us.es/handle/11441/65172

Makleff, S., Garduño, J., Zavala, R. I., Barindelli, F., Valades, J., Billowitz, M., ... \& Marston, C. (2019). Preventing intimate partner violence among young people - a qualitative study examining the role of comprehensive sexuality education. Sexuality Research and Social Policy, 17, 314-325. https://doi.org/10.1007/s13178-019-00389-x

Martínez-Dorado, A., Privado, J., Useche, S. A., Velasco, L., García-Dauder, D., \& Alfaro, E. (2020). Perception of dating violence in teenage couples: A cross validation study in Spain and Colombia. International Journal of Environmental Research and Public Health, 17(18), 6769. https://doi.org/10.3390/ijerph17186769

Mérida, A. P. (2019). Identificación de variables para la valoración del riesgo de violencia contra la pareja en población adolescente. International E-Journal of Criminal Sciences, (14), 1-21. Recuperado de https://dialnet.unirioja.es/servlet/articulo?codi go $=7298465$ 
Ministerio de Salud. República de Colombia (1993). Resolución 8430 de 1993. Recuperado de

https://www.minsalud.gov.co/sites/rid/Lists/B ibliotecaDigital/RIDE/DE/DIJ/RESOLUCIO N-8430-DE-1993.PDF

Muñoz-Rivas, M. J., Graña, J. L., O’Leary, K. D., \& González, M. P. (2007). Aggression in adolescent dating relationships: Prevalence, justification, and health consequences. Journal of Adolescent Health, 40(4), 298-304. https://doi.org/10.1016/j.jadohealth.2006.11.1 37

Organización Mundial de la Salud-OMS (2015). WHO multi-country study on women's health and domestic violence against women. Retrieved from http://www.who.int/gender/violence/who_mul ticountry_study/en/

Palmetto, N., Davidson, L. L., \& Rickert, V. I. (2013). Predictors of physical intimate partner violence in the lives of young women: Victimization, perpetration and bidirectional violence. Violence and Victims, 28(1), 103121.

http://dx.doi.org/10.1891/0886-6708.28.1.103

Paradis, A., Hébert, M., \& Moreau, C. (2020). Mutuality of teen dating violence: Prevalence, Context, risk factors and implications for prevention. In Violence: Probing the Boundaries around the World (pp. 157-184). Brill

Rodopi. https://doi.org/10.1163/9789004429215_011

Peskin, M. F., Markham, C. M., Shegog, R., Baumler, E. R., Addy, R. C., Temple, J. R., ... \& Tortolero, S. R. (2019). Adolescent dating violence prevention program for early adolescents: The $\mathrm{Me} \&$ You randomized controlled trial, 2014-2015. American Journal of Public Health, 109(10), 1419-1428. Retrieved from https://ajph.aphapublications.org/doi/abs/10.2 105/AJPH.2019.305218

Rabin, R. F., Jennings, J. M., Campbell, J. C., \& Bair-Merritt, M. H. (2009). Intimate partner violence screening tools: A systematic review. American Journal of Preventive Medicine, 36(5), 439-445.

http://dx.doi.org/10.1016/j.amepre.2009.01.024
Redondo, J., Inglés, C. J., \& García, K. L. (2017). Papel que juega la edad en la violencia en el noviazgo de estudiantes de la Universidad Pontificia Bolivariana de Bucaramanga. Diversitas: Perspectivas en Psicología, 13(1), 41-54. Recuperado de http://www.scielo.org.co/pdf/dpp/v13n1/1794 -9998-dpp-13-01-00041.pdf

Rey-Anacona, C. A. (2006). Lista de chequeo de experiencias de maltrato en la pareja (Forma A). Manuscrito no publicado.

Rey-Anacona, C. A. (2013). Prevalencia y tipos de maltrato en el noviazgo en adolescentes y adultos jóvenes. Terapia Psicológica, 31(2), 143-154. http://dx.doi.org/10.4067/S071848082013000200001

Rodríguez-Franco, L., López-Cepero, J., Rodríguez-Díaz, F. J., Bringas, C., Antuña, A., \& Estrada, C. (2010). Validación del cuestionario de violencia entre novios (CUVINO) en jóvenes hispanohablantes: Análisis de resultados en España, México y Argentina. Anuario de Psicología Clínica y de la Salud, 6, 45-52. Recuperado de https://idus.us.es/bitstream/handle/11441/1393 9/file_1.pdf?sequence $=1$

Ronzón-Tirado, R. C., Muñoz-Rivas, M. J., Zamarrón-Cassinello, M. D., \& RedondoRodríguez, N. (2019). Cultural adaptation of the Modified Version of the Conflicts Tactics Scale (M-CTS) in Mexican Adolescents. Frontiers in Psychology, 10, 619-628. https://doi.org/10.3389/fpsyg.2019.00619

Rubio-Garay, F., López-González, M. A., Saúl, L. Á., \& Sánchez-Elvira-Paniagua, Á. (2012). Direccionalidad y expresión de la violencia en las relaciones de noviazgo de los jóvenes. Acción $\quad$ Psicológica, 9(1), 61-70. Recuperado de https://www.redalyc.org/pdf/3440/344030769 006.pdf

Rubio-Garay, F., López-González, M. Á., Carrasco, M. Á., \& Amor, P. J. (2017). Prevalencia de la violencia en el noviazgo: Una revisión sistemática. Papeles del Psicólogo, 38(2), 135-147. https://doi.org/10.23923/pap.psicol2017.2831

Shaffer, C. M., Corona, R., Sullivan, T. N., Fuentes, V., \& McDonald, S. E. (2018). 
Barriers and supports to dating violence communication between latina adolescents and their mothers: A qualitative analysis. Journal of Family Violence, 33, 133-145. doi:10.1007/s10896-017-9936-1

Shipley, S. (2018). Intimate Partner Violence: A Systematic Literature Review. Retrieved from https://cornerstone.lib.mnsu.edu/cgi/viewcont ent.cgi? article $=1760 \&$ context $=$ etds

Taquette, S. R., \& Monteiro, D. L. M. (2019). Causes and consequences of adolescent dating violence: A systematic review. Journal of Injury and Violence Research, 11(2), 137-147. doi:10.5249/jivr.v11i2.1061.

Terrazas-Carrillo, E., \& Sabina, C. (2019). Dating violence attitudes among Latino college students: An examination of gender, machismo, and marianismo. Violence and Victims, 34(1), 194-210. doi:10.1891/0886-6708.VV-D-17-00172

Terwee, C. B., Bot S. D. M., de Boer, M. R., der Windt, D., Knol, D. L., Dekker, J., Bouter, L. M., \& de Vet, H. C. W. (2007). Quality criteria were proposed for measurement properties of health status questionnaires. Journal of Clinical Epidemiology, 60(1), 3442.

http://dx.doi.org/10.1016/j.jclinepi.2006.03.012 Toplu Demirtaş, E., Morelli, M., Bianchi, D., Baiocco, R., Chirumbolo, A., Laghi, F., \& Bianchi, D. (2018). Reliability and construct validity of the conflict in adolescent dating relationships inventory-modified (CADRI-M). Retrieved from http://openaccess.mef.edu.tr/bitstream/handle/ 20.500.11779/545/PDR2018_Abstract_etd.pd $\mathrm{f}$ ?sequence $=3 \&$ is Allowed $=\mathrm{y}$

Wolfe, D. A., Scott, K., Reitzel-Jaffe, D., Wekerle, C., Grasley, C., \& Straatman, A. L. (2001). Development and validation of the conflict in adolescent dating relationships inventory. Psychological Assessment, 13(2), 277-293.

http://dx.doi.org/10.1037//1040-3590.13.2.277

Yanez-Peñúñuri, L. Y., Hidalgo-Rasmussen, C. A., \& Chávez-Flores, Y. V. (2019). Revisión sistemática de instrumentos de violencia en el noviazgo en Iberoamérica y evaluación de sus propiedades de medida. Ciencia \& Saúde Coletiva, 24, 2249-2262. http://dx.doi.org/10.1590/1413-

81232018246.19612017 